\title{
Subjetividad, vínculos, narrativa y memoria en la psicoterapia sistémica
}

\section{Una mirada sistémica, ecológica y compleja a la subjetividad}

T a subjetividad ha adquirido especial relevancia en los estudios de Llos fenómenos humanos y sociales en diferentes campos de conocimiento, toda vez que ella ha aparecido como una dimensión propicia para ampliar esos horizontes de la existencia individual y colectiva que permanecieron circunscritos a nociones como las de sujeto e identidad que, como han afirmado diferentes autores, estuvieron por mucho tiempo expuestas a las clásicas dicotomías del pensamiento social así como a los estancos disciplinares que derivaron de ellas. En este sentido, la subjetividad representa un lugar propicio para la revisión crítica de distintos presupuestos disciplinares y, al mismo tiempo, para emprender apuestas de investigación de carácter interdisciplinario y transdisciplinario (Gómez, 2006; Piedrahita, 2006; González, 2012).

En efecto, diferentes campos de conocimiento como la psicología, la historia, la sociología y la antropología, entre otros, le concedieron ascendencia a las nociones de sujeto e identidad, considerándolas indispensables para entender los modos como los individuos o los grupos se hacían al o participaban en el mundo: sujeto e identidad se hicieron 
lugares para afirmar los principios de sujeción, enraizamiento o arraigo de nuestra existencia en el mundo. No obstante, estos campos de conocimiento, expuestos ellos mismos a los efectos de diferentes paradigmas, terminaron exponiendo estas nociones a distintos reduccionismos, sustancialismos o simplificaciones: se consideró al sujeto como una entidad opuesta a lo colectivo o se asumió a lo colectivo como el único sujeto real del mundo social; también se consideró a la identidad como una dimensión inmanente al sujeto o bien como una construcción subsidiaria de la exterioridad de la existencia (cfr. Dubet, 1989; Brubaker y Cooper, 2000; Serna, 2016).

Ahora, se puede afirmar que entre el sujeto y la identidad la dimensión vertebral que fue interpuesta fue la de la conciencia. Mientras unos ámbitos de conocimiento plegaron al sujeto a la conciencia inmediata de sí, otros ámbitos pusieron al sujeto a distancia de conciencia alguna o, en determinados casos, sometido a una situación de falsa conciencia. Se era sujeto en tanto se portaba una conciencia de algo o, por defecto, en tanto se estaba ausente de conciencia alguna — de hecho, esta ambigüedad del sujeto ha acarreado costos altos a las eventuales relaciones entre distintas disciplinas-. La relación entre el sujeto y la conciencia, entendida en términos de distancia, también le dio forma a la identidad. Mientras en unos casos no podía haber identidad que no fuera la del sujeto con conciencia sobre sí mismo, en otros solo podía haberla a condición de que la conciencia del sí mismo quedara excluida en beneficio de una exterioridad colectiva o social. Esta exclusión de los términos, por efecto de las bifurcaciones dicotómicas, no solo llevó a emplazar en lugares separados al sujeto, a la conciencia y a la identidad sino que, más allá, permitieron que ellos fueran revestidos como cosas que serían objetos de conocimiento bien distintos.

Una de las consecuencias evidentes de esta disección, fue que se desvirtuó la complejidad del mundo psicológico: al inscribir en tránsitos escindidos al sujeto, a la conciencia y a la identidad, el mundo psicológico pudo ser fragmentado en dominios separados, no integrados, con lógicas autárquicas, en unos casos reducido a un marco de operaciones y operadores abstraído del mundo social y, en otros, convertido en un simple reflejo de ese mundo social inmediato. Otra 
de las consecuencias de esta disección, fue que al emplazar como dominios separados al sujeto, a la conciencia y a la identidad, se terminó afirmando la predominancia de una cierta heteronomía del sujeto como principio ontológico para comprender el mundo social y para ubicar en esta comprensión el lugar de cada uno de los campos de conocimiento: desde la psicología hasta la sociología y la antropología. De hecho, la crisis de los paradigmas normativos, que partían del presupuesto ontológico de la heteronomía del sujeto, llevó a declarar en distintos lugares la muerte del sujeto (Passerini, 2006).

Ahora, pese a la predominancia que alcanzaron los paradigmas normativos y, con ellos, la creencia en la heteronomía del sujeto, también es cierto que desde diferentes lugares existían antecedentes de propuestas más sensibles a integrar en el sujeto la conciencia de sí y la conciencia del mundo, es decir, más atentos a reconocer el carácter inseparable del sujeto, la conciencia y la identidad. Quizá la noción que mejor sintetiza este lugar alterno sea la de self, esa suerte de yo que emerge allí donde hay otro. El self, del que bien se puede decir que es una subjetividad en estricto sentido, de naturaleza relacional, le confiere especial relevancia a la cuestión del lenguaje: si un horizonte puede desatar las continuidades y discontinuidades entre el sujeto, la conciencia y la identidad este es, sin duda, el lenguaje y no resulta casual que haya sido desde allí, más específicamente desde la narrativa, que se haya planteado una nueva discusión sobre la subjetividad.

No es aventurado señalar que la ascendencia reciente que ha adquirido la subjetividad tiene en medio el efecto de diferentes discursos, entre ellos, de los anclados en los enfoques postestructurales, que encontraron en la subjetividad un horizonte para indagar las capilaridades del poder y para plantear, al mismo tiempo, formas de resistencia y emancipación. No obstante, la invocación recurrente de la subjetividad desde referencias como esta tiende en determinadas circunstancias a desvanecer o difuminar sus especificidades, entre ellas, una que resulta de especial importancia: su relación con la constitución del sujeto en sí, es decir, con la afirmación de unas fronteras psíquicas con el entorno, con la distinción de unos niveles de conciencia, con los modos de relación con el otro, es decir, la subjetividad que involucra la 
individuación, la autonomía y la intersubjetividad (Hernández, 2010). Estos asuntos ceden ante cuestiones de raigambre más estructural como la individualización, que está vinculada de manera estrecha con las improntas de la coacción normativa y con el devenir del poder, con la idea de potencia, en tanto capacidad para hacer en contraposición, y con la subjetivación, en tanto modo de hacerse al mundo.

El enfoque sistémico, ecológico y complejo invita a considerar una ontología de la subjetividad, entendida esta no como una jaula de hierro orquestada por fuerzas inconmovibles, sino como un devenir del sujeto en sus lazos existenciales. Así, este enfoque no disocia la pregunta por la subjetividad de la pregunta por el sujeto, que sigue siendo una preocupación fundamental que sostiene y justifica a diferentes campos de conocimiento, entre ellos, a la psicología.

En consecuencia con esto se entiende la subjetividad como el mundo de lo psicológico, asumido este como el entramado relacional donde coinciden órdenes biológicos, sociales, culturales y políticos en la forma encarnada que es el cuerpo. Las posiciones consecuentes con esta concepción, desafían esa tradición que emplazó a la subjetividad a un ámbito trascendente ajeno a la naturaleza, ora a esa otra tradición que redujo a la subjetividad a ser mera remanente de la naturaleza, extraña a las cualidades peculiares que ofrece la emergencia del socius. La subjetividad desde el enfoque sistémico, ecológico y complejo supone desvirtuar o trascender las oposiciones clásicas (que la reclinan a la interioridad y que de paso la contraponen a la objetividad) para reconocerla desde la intersubjetividad. En este sentido, la subjetividad entraña una dimensión relacional.

Ahora, la reivindicación del carácter sistémico, ecológico y complejo de la subjetividad no supone solo reposicionarla con relación a un discurso de la naturaleza: más allá de eso, supone redefinir lo que se entiende por naturaleza y por la lógica de lo natural. En este sentido, la subjetividad comprende a la naturaleza en un acople que se configura en una auténtica unidad de supervivencia: así, al salir de la idea del "yo" trascendente a espaldas de la naturaleza para involucrar a un yo que surge de la relación con esta, se transforman de manera 
radical viejas cuestiones, entre ellas, una de especial importancia: la conciencia (Bateson, 1993).

Contra la vieja idea que contrapuso la historia a la naturaleza, donde la conciencia afloró como el dominio exclusivo de la racionalidad o como solo un sedimento superficial de la fuerza del inconsciente, nuestro enfoque plantea la posibilidad de una historia natural de la subjetividad, es decir, del mundo psicológico que emerge contextualmente en la vida. Así, se trataría de una subjetividad que entiende al sujeto como "viviente, aleatorio, insuficiente, vacilante, modesto, que introduce su propia finitud. No es portador de la conciencia soberana que trasciende los tiempos y los espacios: introduce por el contrario, la historialidad de la conciencia" (Morin, 1994, p.31). Así entendida, la subjetividad se concibe como una capacidad, como una potencia para formar el sujeto, no en contraposición a un algo que al mismo tiempo modela, sino en tanto emergencia en la vivencia y experiencia del hacer, es decir, desde aquello que Varela (2000) definió como la enacción.

Una vez acá, es evidente que la subjetividad deja de percibirse como una suerte de cosa que se tiene y se conquista de una vez y para siempre, que puede ser rotulada o adjetivada en parcelas separadas, para irrumpir más como una emergencia, esto es, como un proceso surtido por los modos de interacción del ser en el mundo en tanto experiencia, que no sería otra cosa que la vida. La experiencia vivida, que es la interacción del ser con el mundo en tanto sujeto, tiene varias implicaciones, entre ellas, tres que resultan importantes. En primer lugar, supone una ambiciosa redefinición de la identidad, habitualmente capturada por ideas que la reducen a atributos, a posesiones, a pertenencias, es decir, a rasgos inmanentes, que avalan esos fundamentalismos yoicos tan propicios para construir universales conductuales y comportamentales.

La subjetividad, como la entiende el enfoque, supone un nexo entre el "yo" (el I en inglés) y el self (el ME en inglés), esto es, entre la afirmación individual y la construcción social, que deben sus formas a los contextos, siempre cambiantes y múltiples. Precisamente, en este acople sobre lo contingente, se establece la subjetividad como una dimensión relacional, si se quiere, la subjetividad como intersubjetividad, 
que al mismo tiempo trae aparejado al otro, a la alteridad. Así, el proceso de formación del sujeto se entiende como subjetivación pero al mismo tiempo como humanización.

En segundo lugar, relacionado con lo anterior, el emplazamiento de la subjetividad en la experiencia vivida controvierte los esquemas inflexibles que habitualmente estructuran los modos como pensamos procesos como la socialización. Las polaridades recurrentes para entender estos procesos, como aquellas procedentes de ámbitos como la psicología social (internalizaciones, externalizaciones, etc.), se muestran insuficientes para entender la formación de un sujeto que descansa en la inmensidad de posibilidades que ofrece la vida. La vieja idea de los sujetos siempre parciales, que sirvió para establecer desde escalas de desarrollo hasta premisas psiquiátricas, es desafiada por una concepción de la subjetividad que asume las plenitudes del sujeto en los diferentes momentos de su existencia.

Por otra parte, la emergencia de la subjetividad pasa por el cuerpo, una totalidad mediante la cual nos hacemos al mundo. Los modos de hacernos al mundo por vía del cuerpo, es decir, en tanto encarnaciones, redefinen la idea de consciencia: no supone una simple operación cognitiva o cognoscitiva, sino una inscripción existencial. Los modos de hacernos al mundo definen u organizan al self. Las dimensiones culturales deben entenderse como modos de hacerse al mundo, es decir, como mediaciones que al simbolizar el mundo lo plagan de significaciones y sentidos potenciales que se erigen en espacios comprensivos del self.

El síntoma se entiende como una incomprensión o una comprensión limitada de sí que al mismo tiempo supone una pérdida de autonomía. En consecuencia, se entiende la terapia como un ejercicio que abre a la comprensión, que por lo mismo procura la autonomía y que, en consecuencia, es una apuesta por la libertad.

\section{Self, conciencia y vinculación}

Como afirman Francisco Varela y Gregory Bateson, la mente, en cuanto objeto de estudio de la psicología, es una propiedad emergente, una de 
cuyas consecuencias es el Sí mismo. Mi self existe porque me pone en interfase con el mundo. No se puede decir "mi self, mi yo, está aquí, en este componente". El self aparece, se expresa en las dinámicas de interacción, las cuales también se configuran en pautas, dado su carácter retroactivo y repetitivo en el tiempo, propio de los sistemas humanos con historia. Yo soy "yo" en las interacciones, porque ese "yo", sustancialmente, no existe; no está localizado en ningún lugar. Como propiedad emergente, producida por una red subyacente, es un proceso coherente que le permite al sistema en el cual existe - un self específico-, interactuar en ese nivel de realidad, es decir, con otros yoes o identidades de la misma especie.

El "yo" opera gracias a la conciencia, entendida como la capacidad que le permite al sujeto dar cuenta y darse cuenta de lo vivido en el mundo externo y en su mundo interno. Se habla de mundo interno en el sentido de que existe porque cada individuo lo testimonia, sin que los demás tengan acceso directo a ese mundo subjetivo, a no ser que el sujeto comunique sus contenidos y a veces sus procesos, a través del lenguaje y de la interacción. Se podría por tanto decir que el self y la conciencia del sujeto operan en constantes procesos de introspección y de interacción.

Porque la conciencia no es un recipiente donde se almacenan contenidos o recuerdos como informaciones fijas e inmutables como en un archivo bloqueado de computador; la conciencia es el proceso de darse cuenta y por tanto opera como un dispositivo dinámico que configura permanentemente la experiencia del sujeto, en relación con los demás procesos que constituyen y le dan vida a su mundo subjetivo, como son los procesos cognitivos, afectivos y motivacionales; en esos procesos participa el cuerpo con la anatomía y la fisiología propias de la especie homo sapiens, las capacidades senso perceptivas, las aptitudes intelectuales, la memoria y los llamados impulsos o instintos que activan al individuo en función de su supervivencia y sus deseos.

La conciencia en cuanto proceso, es un proceso paradójico, porque en un mismo instante no puede darse cuenta y a la vez ser consciente de que se da cuenta. En este aspecto, la conciencia humana es limitada y circunscribe el self a un determinado contexto de experiencias. 
Por eso, aunque el individuo está sujeto a infinidad de estímulos, la capacidad de la conciencia, como diría el cibernetista Miller (1955), solo puede procesar siete más o menos dos bits de información en un momento dado. Es allí donde los procesos de interacción participan en la emergencia del self, porque esa configuración adquiere su forma coherente en el tiempo, gracias a que también es retroalimentada en las relaciones con los demás. Esas relaciones obedecen a complejas codificaciones que conjugan los distintos sistemas de simbolización creados por los hombres, como son, en términos de los operadores de la vinculación, los sistemas de significación y los sistemas de organización de la interacción.

En otras palabras, como lo concibe E. Morin (1994), el self emerge como una configuración psíquica que conjuga los determinismos y el azar que corresponden a la organización biológica de la especie y a la organización social y cultural en los pequeños nichos de interacción como la familia y los grandes contextos formalizados en las sociedades que son las instituciones. Ese sistema complejo especie-psiquismo-sociedad-cultura está atravesado por el hilo del tiempo flecha y por el tiempo circular que constituye patrones, de modo que en el interjuego de esas dos modalidades del tiempo van danzando la permanencia y el cambio, la identidad y la construcción cambiante de ella misma.

Es en este contexto que puede afirmarse que el self surge en la interacción y conserva su coherencia como un mecanismo adaptativo que opera sobre un principio de economía para la supervivencia y que organiza las experiencias del sujeto de una forma diferencial que constituye su identidad. Ese principio de economía para la adaptación no aplica solo al sujeto, sino a todos los que participan en la interacción, quienes a su vez necesitan estructurar la complejidad de la vida y contener la incertidumbre absoluta que habría si cada momento se viviera en su total emergencia novedosa.

Eso no significa que los demás estímulos que circulan en cada instante desaparezcan, sino que se pueden conservar gracias a la memoria como dispositivo y como proceso, en instancias conocidas como subconsciente e inconsciente, las cuales en apariencia operan con base 
en códigos y convenciones distintas a las que permiten la vinculación entre sujetos.

Se diría entonces que esas convenciones y códigos que circulan en el mundo de la conciencia, adoptan la forma de sistemas de interacción (pautas de relación, rituales, procedimientos) y sistemas de significación (creencias, mitos, epistemes), precisamente para hacer de la interacción humana un nivel de realidad constante en función de la adaptación y la supervivencia de la especie, la sociedad y la cultura.

En ese contexto, el malestar de los sujetos y de los grupos humanos, surgiría como una suerte de asincronía entre los niveles de realidad interactuantes, entendiendo que cada una de esas dimensiones o niveles aporta en forma específica en cada caso, y por lo tanto, también los problemas que se convierten en motivo de consulta en la psicoterapia, son a su vez una configuración compleja que opera en forma restrictiva de los procesos vitales inherentes a cada ámbito — sujeto, grupo, sociedad-. En otras palabras, la llamada "psicopatología” sería una configuración compleja que frena la adaptación y la existencia autónoma de los sujetos ecodependientes y en su surgimiento siempre participan las dimensiones biológicas, subjetivas e interaccionales.

La psicoterapia sistémica compleja sería así un dispositivo para activar los estancamientos de los sujetos en sus contextos vitales, algunas de cuyas características serían las siguientes:

- $\quad$ Si bien reconoce la presencia de todas las dimensiones de la vida en cada situación problema, su dominio de acción es la interacción que circula en la vinculación entre consultantes y terapeutas.

- Esa relación se constituye en sí misma en un sistema complejo, dentro del cual participan simultáneamente “yoes”, conciencias, sistemas de interacción y sistemas de significación.

- Los procesos de vinculación terapéutica son en sí mismos dispositivos de transformación y utilizan como agentes de cambio esos mismos ingredientes - “yoes”, conciencias, sistemas de interacción y sistemas de significación-. Es decir, se cuestionan los "yoes", se expanden las conciencias y se perturban 
los sistemas de interacción y de significación en función de las finalidades deseadas por los consultantes y acordadas con los terapeutas, en coherencia con la concepción de la psicoterapia como un proceso estructurado y contextualizado en tiempo y espacio.

- El acceso a los “yoes”, las conciencias y los sistemas de significación y de interacción se hace mediante conversaciones entre consultantes y psicoterapeutas que se organizan con la lógica propia de la psicoterapia. En esas conversaciones se observan interacciones, se hacen relatos, reflexiones, cuestionamientos, confrontaciones, metáforas, se cuentan historias, etc., con la pretensión de generar nuevas formas de organización de las subjetividades y las interacciones.

En esta perspectiva, la memoria es uno de los procesos cognitivos encarnados, en interacción permanente con todos los demás procesos psicológicos (senso percepción, inteligencia, razonamiento; emociones, deseos e impulsos; finalidades y motivaciones). Es un dispositivo que organiza la información procedente de todos los demás procesos psicológicos y neurofisiológicos en interacción entre sí y con el entorno, en forma de recuerdos, más o menos conscientes, que pueden expresarse en forma más o menos estructurada, desde imágenes y sensaciones aisladas hasta relatos elaborados. Parece que es aún un misterio la forma como se produce esa organización, la cual, de todos modos aplica mecanismos de selección, clasificación, inclusión, exclusión (olvido) y conexión de información, obedeciendo a su vez a los principios operadores de la memoria y del mundo cognitivo como sistema. Esto significa que más que un recipiente que guarda datos, la memoria es un proceso que trae a la conciencia informaciones que se van activando y conectando al ser nodos de una red neuro-subjetiva-social (parece que también eléctrica), como se van encendiendo las luces de un árbol navideño según una programación previa. El problema para los psicoterapeutas y para todas las personas, es que desconocemos la programación de esa red. 


\section{Sobre la narración como dominio}

De entrada se puede afirmar que la narración es el dominio que emerge de la vinculación y permite acceder a la subjetividad: la condición pragmática del lenguaje narrado que es constitutivo del self. En consecuencia con esto también se puede afirmar que el relato constituye un ámbito de organización de lo psicológico y, al mismo tiempo, un mecanismo adaptativo. La narración por medio del relato formaliza la comunicación.

Las prácticas narrativas son una forma comunicacional presente en los diversos ámbitos de la vida humana y específicamente en la psicoterapia tiene que ver con los siguientes referentes:

- Las prácticas narrativas son una forma material de conversar en torno a acontecimientos y experiencias de la vida, en donde el uso del lenguaje, verbal, no verbal, simbólico o pictórico, dramático o retórico es constitutivo ontológico de esa comunicación. Dichas formas de las expresiones humanas, revisten un carácter de interpretación subjetiva e intersubjetiva de experiencias vividas por los inter-pretantes o narradas en formas de relatos culturales por familias o comunidades, todo lo cual nos advierte del carácter intersubjetivo de toda narrativa, la cual puesta en marcha en un contexto de psicoterapia sistémica enfatiza en la posibilidad de establecer una "hermenéutica experiencial", con-vivencial y solidaria.

- La narración se pone en curso en la interacción entre dos o más personas y avanza durante el curso de la conversación definida como psicoterapéutica; esta narración emerge en este contexto interaccional con un sentido contextual, auto-referencial y simbólico que le confiere diversidad de órdenes de significado al encuentro en sí mismo, a los sistemas de relaciones evocados donde emerge el self en interacción en sus diferentes configuraciones; en este sentido, permite preguntarse quién habla, desde dónde habla y qué sentido tienen ese relato y esa voz en el escenario del encuentro. La importancia de esta narrativa radica en que plasma el inter juego de las subjetividades 
presentes y ausentes, las cuales son, en todo caso, referentes contextuales y meta-contextuales que le confieren sentido a la trama humana en curso.

- Este dominio narrativo conversacional se establece en el curso del encuentro terapéutico, como un dispositivo organizador de una trama humana que sufre un tratamiento por parte de los actores y que intenta de alguna manera resolverse, de modo que se encarna el sentido en las subjetividades e intersubjetividades que se ponen en juego como una condición fundamental para comprender la dinámica de los modos y formas de vinculación entre los sistemas humanos evocados en el curso del proceso psicoterapéutico.

- Los elementos constitutivos de estas prácticas narrativas, suponen una acción evocadora de experiencias en primera persona, lo cual pone en juego las percepciones e interpretaciones del "sí mismo" y de "los otros", que están presentes o que estando ausentes hacen presencia en el escenario de la conversación, es decir confieren un valor en y a los acontecimientos vividos por los personajes presentes en las narraciones. De hecho estas acciones implican una percepción subjetiva, personal e interpersonal de los acontecimientos narrados y vividos por los actores. En ese mismo orden, el sentir de tales narraciones invita a que los narradores se dispongan de un modo personal en las interacciones que contextualizan el acto de narrar. De manera recíproca el acto de narrar incide sobre el contexto para posicionar a los testigos y posibilita la configuración de un co-narrador que puede incidir en el decurso de las vicisitudes de la conversación narrativa.

- Las prácticas narrativas se refieren a los relatos que se evocan en una conversación situada en un contexto interaccional concreto, constituido con la intención de resolver un dilema, problema o conflicto humano. La conversación narrativa es una modalidad que permite atribuirle sentido y significado a las interacciones en curso en el proceso de la conversación definida como psicoterapéutica, la cual resulta de procesos abstractos, complejos 
y subjetivos que organizan la experiencia en episodios temporalmente significativos para los participantes en el encuentro.

- Esta forma de comunicación definida como prácticas narrativas, señala la posibilidad de que los seres humanos re-editemos las formas de vinculación presentes en las historias y relatos de vida, gracias no solamente a compartir los significados evocados en los relatos, sino a crear nuevas versiones en torno a los modos de vivir. Las posibilidades que engendra el mecanismo de la re-flexividad instaurado en la interacción conversacional están dadas por la expansión de la subjetivad desplegada. Al compartir dialógica y reflexivamente nuestra experiencia ponemos en marcha los dispositivos conversacionales y narrativos para crear nuestras identidades personales y colectivas. Estas identidades experimentan su comprensión en las valoraciones que hacemos acerca de quién narra, cuándo narra y para quién narra, que permite comprender y explicar la dinámica de los modos de vinculación en los sistemas humanos.

- Las prácticas narrativas como dispositivo de convivencia humana, nos permiten dar cuenta de numerosos procesos mentales y sociales, subjetivos e intersubjetivos que caracterizan a las personas, parejas, familias y comunidades, en un momento dado, brindando a los consultores y psicoterapeutas un dominio fenomenológico para explicar y comprender las demandas, problemas y conflictos humanos que requieren soluciones.

- Las prácticas narrativas, definidas como meta-sistemas de significación inherentes a todo sistema humano exceden para su comprensión a las narrativas convencionales de la texto y socio-linguística en tanto que en las “prácticas narrativas” el relato que se pone en marcha, es un dispositivo para comprender las interacciones en curso en la conversación psicoterapéutica; como objeto semántico y semiótico es sometido a connotaciones, interrogado e interpretado en la experiencia inmediata de las interacciones en transformación, por lo tanto como mecanismo organizador de la experiencia emerge en el contexto de la intersubjetividad humana. 
- En las prácticas narrativas, al establecer una relación causal circular entre el relato y el contexto de evocación sistémicamente orientado, se comprende la emergencia de los significados y del sentido de las interacciones, de modo que una acción conjunta, genera una intersubjetividad creadora. No se trata de un estudio de significados y formas, sino de la creación de una producción social de sentidos. No se parte de a priori, sino que con las formas del lenguaje se genera una práctica social donde surge la comprensión del acontecimiento humano.

- Un texto narrativo conversacional como forma básica está constituido por narraciones de la vida cotidiana, incluye los chistes, cuentos populares, leyendas, novelas, biografías, cuentos, y estos textos como dispositivos conversacionales, nos permiten interpretar la experiencia; la importancia está dada por la posibilidad de ser utilizado como herramienta que viabiliza la construcción de un contexto interactivo que por lo demás podemos llamar o definir como contexto narrativo, en tanto intersubjetivamente están situadas en un proceso interaccional concreto.

- El tránsito de una narrativa textual a un contexto narrativo está fundamentado en que el texto del sujeto puede ser interpretado con el reconocimiento pleno de su voz en la interacción, es decir en la narrativa conversacional. El acto narrativo se legitima por la identidad de las voces en el contexto de la interacción, la cual garantiza la emergencia subjetiva e intersubjetiva de los diversos significados posibles de ser creados en el decurso de la conversación. En síntesis, cuando hablamos de prácticas narrativas, evocamos de hecho la percepción subjetiva e intersubjetiva en contexto y por tanto garantizamos el desarrollo de una trama humana que posibilita el desenlace en el decurso de la conversación.

- Sabemos por todas las investigaciones en el campo de la narrativa que la acción de contar historias y relatos está íntimamente ligada con un carácter temporal de la existencia humana, en tanto que el tiempo físico se hace humano cuando se articula como narración y cuando esta narración cuenta acerca de la existencia 
temporal de la condición humana. En este orden de ideas no se trata de recordar eventos ni de representarnos acontecimientos sino de vivir la experiencia de narrar y esta experiencia se torna como un dispositivo para reconfigurar todos los acontecimientos vividos y hacerlos presentes en la conversación; por lo tanto se trata de una lógica de la interacción humana, la cual, al evocar la subjetividad anticipa una reconfiguración de las formas de vivir la vida humana y es así una prefiguración práctica y una reconfiguración concreta de las posibilidades de reencarnar la vida en el marco de nuevos significados. Todo lo anterior es posible gracias al mecanismo autorreferencial y heterroreferencial de los sistemas en interacción, gracias a esta capacidad reflexiva que pone en marcha la reconfiguración encarnada de nuevos relatos.

\section{Memoria y subjetividad}

Nuestra concepción de la subjetividad desafía a la memoria: la cuestiona en cuanto esta tiene de cosificación, para buscarla en cuanto esta tiene de procesual y relacional. La idea de la memoria como cosa es, precisamente, una consecuencia de ciertas lecturas de la naturaleza, en particular del naturalismo que supuso que los procesos vitales tenían emplazamientos específicos en el orden físico de los cuerpos, en la estructura cerebral, de la cual la estructura mental era apenas una expresión epifenoménica. Fuera del orden físico de los cuerpos que pertenecía a la naturaleza entraban en juego las dimensiones de la existencia que pertenecían propiamente a la sociedad. La memoria, así, era en lo fundamental el resultado de unas estructuras y funciones corporales y, cuanto la sociedad pudiera hacer por ella, era accidental, superficial o meramente contingente o supeditado a unas estructuras que aunque pudieran ser comparadas con las estructuras naturales, no obstante tenían sus formas o leyes propias.

En oposición a esta concepción de la memoria se levantaron diferentes posturas: desde las filosofías espiritualistas que no le concedían a la materia ni a los materialismos la potestad sobre lo que se consideraban las facultades superiores del espíritu, cual más la memoria, 
hasta el psicoanálisis que convirtió a esta en huella de una estructura inconsciente irreductible a la materia. No fue casual por ello que la memoria se constituyera en uno de los objetos privilegiados del conflicto entre la filosofía y la ciencia a finales del siglo XIX y que, en algunas tradiciones, como la francesa, los primeros derroteros para establecer una psicología científica estuvieran soportados en un esclarecimiento metódico de la memoria como objeto de conocimiento científico, un debate que se muestra de manera excepcional en autores como Dugas (1894 y 1917).

De cualquier manera, la oposición entre naturalismos y espiritualismos o, más recientemente, entre psiquismos y sociologismos, convirtió a la memoria en un objeto disperso, simplificado o reducido, entre distintos estancos disciplinares. En efecto, la memoria es un dominio de la vida especialmente expuesto a las compartimentaciones del conocimiento provocadas por la especialización disciplinar. Una de las consecuencias de estas compartimentaciones es la exposición de la memoria a una serie de dicotomías que, establecidas desde las distinciones arbitrarias de distintos campos de conocimiento, han terminado no obstante constituidas en atributos naturales de la memoria en sí: memoria individual y memoria colectiva, memoria social y memoria histórica, etc. En este sentido, las distinciones artificiales que fueron impuestas para simplificar los modos de conocimiento terminaron constituidas en atributos naturales de los objetos de conocimiento como tales. De este modo, bajo formas dicotómicas, la memoria es revertida a la vida pero al costo de la vida y la memoria: ellas quedan expuestas a unas distinciones que no existen en la inconmensurabilidad de la vida, obligadas a unas relaciones de espacio y tiempo que les son extrañas, que convertidas en variables objetivas tienen la capacidad de desmantelar a la experiencia y que, con ello, hacen insustancial a la narración. La memoria así se erige en una simple ciencia auxiliar de unas viejas formas de hacer historia (incluida la clínica), preocupada solo por sus aspectos puramente cognoscitivos, obligada solo a representaciones y, en determinadas circunstancias, asediada por el riesgo de la falibilidad. Es de este modo como estas formas dicotómicas reducen cualquier posibilidad de la memoria al esclarecimiento de situaciones fácticas, 
fuera de las cuales no puede ser memoria sino apenas fantasía o imaginación (síntoma o padecimiento o, en los mejores casos, asunto de campos disciplinares ajenos a la clínica).

Estas concepciones permiten instrumentalizar la memoria: inscrita en la naturaleza como cosa en sí o extrapolada en la sociedad como acción o práctica para hacer o ejecutar, la memoria es un dominio propicio para construir toda suerte de discursos esencialistas sobre los acontecimientos humanos, sobre la naturaleza de las vicisitudes, sobre los modos de afrontamiento y sobre las maneras como los individuos, los grupos o las sociedades elaboran sus procesos de transformación y cambio. De esta instrumentalización surgen los discursos recurrentes sobre el determinismo del trauma, sobre el recuerdo como catarsis, sobre el olvido como anatema, sobre la verdad como imperativo, sobre la memoria como garantía de no repetición. Todos estos discursos, recurridos y recurrentes, tienen el problema que habitualmente no explican cómo semejantes cosas son posibles más allá de una suerte de potencia de la memoria en sí (Serna, 2012).

Las concepciones dicotómicas y simplificadoras de la memoria son confrontadas por una concepción que entiende a la naturaleza como emplazamiento de la vida en cuanto esta tiene de relacional, de procesual, de emergente y de contextual, esto es, una idea de la naturaleza anclada a la complejidad de la vida en interacción, inseparable de la experiencia y del lenguaje, no escindida de la sociedad (con todo lo que ella representa en términos políticos y culturales), donde la estructura y la función son entendidas como emergencias provisionales de determinados estados de un sistema. La memoria, así comprendida, se presenta en términos ontológicos como una adaptación y en términos epistemológicos como un proceso, de tal suerte que cualquier pretensión de recuerdo y olvido supone siempre, en cualquier circunstancia, el contexto (una forma provisional de exposición del proceso, una organización en espacio y tiempo de la adaptación).

En consecuencia con lo anterior, una concepción compleja de la memoria parte del principio de que ella es un principio organizador de la vida que se debate en órdenes o niveles de complejidad, donde la posibilidad de retención, de repetición, de reiteración o de innovación 
no solo se deben a unas formas de organización en un momento determinado sino que, en ciertas circunstancias, ellas no solo sirven para recordar sino, también, para olvidar, dejar el guión o poner entre paréntesis, porque se daña la redacción contra lo que refieren tantos emprendimientos en pos de la memoria, no hay mejor forma de olvidar que repetir o reiterar. En los sistemas humanos, este principio de organización de la vida está profusamente vinculado no solo a los cuerpos sino a las narraciones, esto es, a los modos prácticos de ocupar al mundo y a las formas de referir estos modos de ocupación para los otros: es por este medio, del cuerpo que se hace al mundo y haciéndolo se refiere a él, que la memoria entra a ser parte de una suerte de consciencia encarnada, una consciencia que no está supeditada a ningún psiquismo primordial, a un yo aislado, pero tampoco a una conciencia o representación colectiva. Con esto, con esta memoria entendida como conciencia encarnada, se pueden recuperar una serie de órdenes o dimensiones que quedaron marginadas por los enfoques puramente cognoscitivos de la memoria o que fueron convertidos en recursos puramente especulativos de los mercados de la memoria, como por ejemplo, las emociones, los afectos y los sentimientos.

Así, por medio de esta concepción compleja de la memoria, es posible revisitar lugares que las disciplinas convirtieron en inamovibles de las explicaciones disciplinares, entre ellas, nociones como las de hábito, costumbre, recuerdo, olvido. Con esta concepción, es evidente que ningún trabajo de la memoria puede tener lugar fuera de las vinculaciones humanas y que ningún proceso de reparación asociado a la memoria puede proceder al margen de lo que suceda con las vinculaciones humanas. Es allí, hacia ese lugar, hacia donde deben apuntar los enfoques de la memoria decididos o convencidos de que ella es útil para la reconstrucción del mundo social.

Precisamente, nada mejor para reconocer esta naturaleza compleja de la memoria que el escenario de la consulta, donde esta no se entiende como un puro lugar instrumental para el relato sino, más allá, como el ordenamiento o el horizonte donde el relato se manifiesta en tanto expresión encarnada de unas formas de organización, inseparable de los mitos, los ritos y las epistemes. 